\title{
Bayesian Stable Mixture Model of State Densities of Generalized Chua's Circuit
}

\author{
F. Acar Savaci* and Serpil Yilmaz ${ }^{\dagger}$ \\ Izmir Institute of Technology, \\ Department of Electrical and Electronics Engineering, \\ Gulbahce, Urla 35430, Izmir, Turkey \\ *acarsavaci@iyte.edu.tr \\ ${ }^{\dagger}$ serpilyilmaz@iyte.edu.tr
}

Received April 16, 2014; Revised September 26, 2014

\begin{abstract}
In this paper, the probability density functions (PDFs) of the states of Generalized Chua's Circuit (GCC) have been modeled by Finite Mixture $\alpha$-Stable $(\mathrm{FM} \alpha \mathrm{S})$ distributions which is a Bayesian mixture model of $\alpha$-stable distributions and it provides semiparametric characterization for the distributions of multiscroll chaotic attractors. Fully Bayesian approach has been applied to estimate the mixture parameters of multimodal distributions corresponding to the multiscroll chaotic attractors.
\end{abstract}

Keywords: Bayesian stable mixture model; Finite Mixture $\alpha$-Stable $(\mathrm{FM} \alpha \mathrm{S})$ distributions; semiparametric modeling; probability density functions; Generalized Chua's Circuit; multiscroll chaotic attractors; multimodal distributions.

\section{Introduction}

The density estimation is an important subject for the characterization of the observed data obtained from real physical systems. By the estimated parameters, valuable information can be extracted from the data. In statistical applications, Finite Mixture (FM) distributions have been of great interest to model heterogeneous data in various fields such as astronomy, biology, economics, engineering, and genetics [McLachlan \& Peel, 2004; Gelman et al., 2013]. In real world applications, the distributions of the dynamics are complicated and the special distributions like Gaussian, Poisson are not sufficient for parametric estimation of such complicated distributions [Zong, 2006; McLachlan \& Peel, 2004]. Since many physical phenomena such as stochastic disturbances in power systems [Mei et al., 2011], underwater acoustics, atmospheric noise [Nikias \& Shao, 1995; Janicki \& Weron, 1993] show impulsive characteristics; then the related statistics might have heavy-tailed distributions.
Such an impulsive behavior with heavy-tailed distributions have been modeled by Stable Distributions (referred to as alpha-stable ( $\alpha$-stable) distributions) which also allow for modeling data with skewed distributions [Samoradnitsky \& Taqqu, 1994]. Furthermore, if such data have multimodality characteristics then Finite Mixture of $\alpha$-stable (FM $\alpha \mathrm{S}$ ) distributions introduced in [Casarin, 2004] could be used for modeling.

The attractors of chaotic dynamics have heavytailed distributions because at the boundaries of the corresponding trajectories the speed of the trajectories comparatively decelerate thus the possibility of occurrence of the points at the boundaries increase. This fact inspired us to model the chaotic dynamics by $\mathrm{FM} \alpha \mathrm{S}$ distributions, and thus heavy-tailness. Further to this observation since $\mathrm{FM} \alpha \mathrm{S}$ distributions are a more general class of distributions including Gaussian distributions, FM $\alpha \mathrm{S}$ distributions have become more convenient in statistical modeling instead of using Parzen estimator given 
in [Günel, 2004; Gunel \& Savaci, 2005a; Günel \& Savaci, 2005b]. To the best of our knowledge, the chaotic dynamics have been modeled firstly in this paper by Bayesian mixture of $\alpha$-stable distributions by applying to the state densities of Generalized Chua's Circuit (GCC). The GCC presented in [Suykens et al., 1997; Suykens \& Vandewalle, 1993] have been deliberately chosen because while each cluster in GCC can be modeled by single $\alpha$-distributions, multimodality characteristics of multiscroll attractors can be modeled by $\mathrm{FM} \alpha \mathrm{S}$ distributions.

Though state densities of GCC have been already estimated by a nonparametric approach using B-splines in [Savaci \& Güngör, 2012] as an alternative method to the Parzen estimator given in [Günel, 2004; Günel \& Savaci, 2005b]; however, the newly proposed $\mathrm{FM} \alpha \mathrm{S}$ distributions provide semiparametric characterization for the distributions of the attractors of the chaotic dynamics of GCC which are indeed stationary distributions since the transients have been ignored in the estimation procedure.

$\alpha$-stable distribution can be considered as a semiparametric model because although $\alpha$-stable distributions do not have closed-form expressions, the characteristic function of a random variable with stable distribution is described by four parameters as given in Sec. 2.1. Such finite mixture of $\alpha$-stable $(\mathrm{FM} \alpha \mathrm{S})$ distributions can be considered as a Bayesian semiparametric model. This model is called Bayesian because in this hierarchical model, posterior distributions and $\alpha$-stable distributions in the final mixture model are evaluated via prior distributions chosen from the known distributions such as Normal, Gamma, Uniform distributions.

In modeling $\mathrm{FM} \alpha \mathrm{S}$ distributions if the number of mixtures (clusters) are a priori unknown then the number of clusters is also to be estimated which is a very hard problem. The mixture model of Normal Distributions for the case of unknown number of mixtures in the Bayesian framework is given in [Green, 1995; Richardson \& Green, 1997; Webb \& Copsey, 2011] with Reversible Jump Markov Chain
Monte Carlo (RJMCMC) method to solve the Model Selection Problem [Andrieu et al., 2001]. However, for the case of known number of mixtures (\# of clusters known) the mixture model of $\alpha$-stable distributions has been presented in [Casarin, 2004; Lombardi, 2007]; furthermore, in [Salas-Gonzalez et al., 2009] the number of mixtures is assumed to be unknown (\# of clusters unknown) which is the case usually encountered in real applications. In order to estimate the number of mixtures, RJMCMC method have been applied in [Salas-Gonzalez et al., 2009].

The rest of this paper is organized as follows: In Sec. 2.1, $\alpha$-stable distributions have been briefly introduced and in Sec. 2.2, the Bayesian algorithm for the estimation of $\mathrm{FM} \alpha \mathrm{S}$ distributions has been presented compactly, but yet, the content is sufficient for applying to any data obtained from dynamical systems. In Sec. 3, using the Bayesian algorithm the state densities of GCC have been estimated by $\mathrm{FM} \alpha \mathrm{S}$ distributions and the distribution of multiscrolls of GCC has been characterized by the parameters of the estimated FM $\alpha \mathrm{S}$ distribution. Assuming that the number of mixtures is known (\# of clusters known) the distributions of multiscrolls have been modeled by $\mathrm{FM} \alpha \mathrm{S}$ distributions using the Bayesian algorithm through Secs. 2.2.1-2.2.2; whereas assuming that the number of mixtures is unknown (\# of clusters unknown) then the Bayesian algorithm has been applied through Secs. 2.2.1-2.2.3. In Sec. 3.5, the comparison of $\mathrm{FM} \alpha \mathrm{S}$ modeling with the parametric Gaussian Mixture Model (GMM) and Parzen estimator has proved the superiority of $\mathrm{FM} \alpha \mathrm{S}$ modeling.

\section{Bayesian Stable Mixture Model}

\section{1. $\alpha$-stable distributions}

Although there is no analytical expression for $\alpha$ stable density functions, the characteristic function of a random variable $X$ which has a stable distribution can be described as [Samoradnitsky \& Taqqu, 1994; Nikias \& Shao, 1995]

$$
\varphi(w)= \begin{cases}\exp \left\{-|\gamma w|^{\alpha}\left[1-i \beta \operatorname{sign}(w) \tan \left(\frac{\pi \alpha}{2}\right)\right]+i \mu w\right\} & \text { for } \alpha \neq 1 \\ \exp \left\{-|\gamma w|\left[1+i \beta \operatorname{sign}(w) \frac{2}{\pi} \log (|w|)\right]+i \mu w\right\} & \text { for } \alpha=1\end{cases}
$$


where $\operatorname{sign}(w)$ is signum function and $0<\alpha \leq 2$, $\beta \in[-1,1], \gamma \in \mathbb{R}_{+}$, and $\mu \in \mathbb{R}$.

A stable distribution is characterized by four parameters: $\alpha, \beta, \mu, \sigma$ and denoted by $S_{\alpha}(\sigma, \beta, \mu)$. The characteristic exponent denoted by $\alpha$ measures the tail thickness of the distribution (smaller $\alpha$ implies heavier tails i.e. more impulsive behavior). The skewness parameter $\beta$ measures the symmetry of the distribution where $\beta=0$ refers to symmetric distribution, $\beta>0$ right-skewed distribution and $\beta<0$ left-skewed distribution. $\mu$ is a location parameter and $\gamma$ is the dispersion parameter which determines the spread of distribution around its location parameter $\mu$ as the variance of a Gaussian distribution determines the spread around the mean. In fact the Gaussian, Cauchy, and Levy distributions are special cases of the $\alpha$-stable distributions with $(\alpha=2),(\alpha=1, \beta=0)$, and $(\alpha=1 / 2, \beta=1)$, respectively.

In the $\mathrm{FM} \alpha \mathrm{S}$ distributions model, the numerical approximation of $\alpha$-stable density functions has been evaluated [Nolan, 1997] by the inverse Fourier transform of the characteristic function of $\alpha$-stable distributions given in Eq. (1) as

$$
f(y ; \alpha, \beta, \gamma, \mu)=\frac{1}{2 \pi} \int_{-\infty}^{\infty} e^{-j w y} \varphi(w) d w .
$$

\subsection{Bayesian algorithm for estimation of mixtures of $\alpha$-stable distributions}

For single-mode data, the methods for parameter estimation in the special case of symmetric stable distributions $(S \alpha S)$ have been introduced in [Ma \& Nikias, 1995] whereas Kuruoglu [2001] has developed estimators for the parameters of skewed $\alpha$-stable distributions. For multimode signals usually observed in physical systems a fully Bayesian approach has been applied for the estimation of $\mathrm{FM} \alpha \mathrm{S}$ distributions [Casarin, 2004]. When a priori knowledge for the data is available, the parametric modeling for the estimation is preferred to nonparametric modeling [Scott, 2009]. Bayesian methods provide a probabilistic model via estimating posterior distributions of the unknown parameters that characterize the data using prior distributions [Gelman et al., 2013; Webb \& Copsey, 2011]. Prior parameters are all drawn independently from the highly encountered distributions such as normal, gamma, uniform distributions. Such a selection provides weak information about the prior parameters
[Richardson \& Green, 1997]. Posterior distributions are computed using the Monte Carlo Markov Chain (MCMC) method in a Bayesian framework.

The stable distributions mixture model for the data vector $\mathbf{y}$ is given by

$$
p_{Y}(\mathbf{y})=\sum_{j=1}^{k} w_{j} f_{j}\left(\mathbf{y} \mid \boldsymbol{\theta}_{j}\right)
$$

where $p_{Y}(\mathbf{y})$ is the probability density function of the vector observations $\mathbf{y}=\left[\begin{array}{lllll}y_{1} & y_{2} & \cdots & y_{m} & \cdots\end{array}\right.$ $\left.y_{N}\right]$ and $w_{j} \in \mathbb{R}_{+}$is the mixture proportion such that $\sum_{j=1}^{k} w_{j}=1$ where $j=1,2, \ldots, k$ denotes the clusters (subpopulations) from which the data are randomly drawn. The likelihood function $f_{j}\left(y_{m} \mid \boldsymbol{\theta}_{j}\right)$ is the density of the $m$ th observation $y_{m}$ knowing that $m$ th observation is from the $j$ th cluster where $\boldsymbol{\theta}_{j}=\left[\begin{array}{llll}\alpha_{j} & \beta_{j} & \gamma_{j} & \mu_{j}\end{array}\right]$ is the parameter vector for the $j$ th cluster. The likelihood functions throughout the Bayesian algorithm have been numerically evaluated using Eq. (2).

Let $z_{i}$ be the allocation variable which is supposed independently to be drawn from the distribution given by Eq. (3) and $z_{i}=j$ denotes that observation $y_{i}$ is drawn from the $j$ th cluster of the mixture

$$
p\left(z_{i}=j\right)=w_{j} \quad \text { for } j=1,2, \ldots, k .
$$

Assigning the allocation vector $\mathbf{z}=\left[\begin{array}{ll}z_{1} & z_{2}\end{array}\right.$ $\left.\begin{array}{llll}\cdots & z_{i} & \cdots & z_{N}\end{array}\right]$ then the probability of the $i$ th observation being from the $j$ th cluster is given by $f_{j}\left(y_{i} ; \boldsymbol{\theta}_{j}, \mathbf{z}\right)$ i.e.

$$
y_{i} \mid z \sim f_{j}\left(\cdot \mid \boldsymbol{\theta}_{j}, \mathbf{z}\right) .
$$

The weight vector $\mathbf{w}$, the component parameters vector $\boldsymbol{\theta}_{j}$ and the number of allocations (i.e. the number of clusters) $k$ are the unknown variables which are to be estimated. With the Bayesian approach the posterior probability distribution of unknown variables can be estimated by the given observation $\mathbf{y}$ and the prior distribution of $k, \mathbf{w}$ and where $\boldsymbol{\theta}=\left[\begin{array}{llll}\boldsymbol{\theta}_{1} & \boldsymbol{\theta}_{2} & \cdots & \boldsymbol{\theta}_{k}\end{array}\right]$. The selection of prior information has been presented in the following sequel [Richardson \& Green, 1997; SalasGonzalez et al., 2009].

\subsubsection{Selection of the priors for $k, \mathbf{w}, \boldsymbol{\theta}$}

(i) Selection of prior information for the number of allocations $k$ : 
The number of allocations $k$ is priorly drawn from the uniform distribution

$$
p\left(k \mid k_{0}\right)= \begin{cases}\frac{1}{k_{0}-1} & 1 \leq k \leq k_{0} \\ 0 & \text { elsewhere }\end{cases}
$$

(ii) Selection of prior information for weights w: The prior distribution for the weights $\mathbf{w}=\left[w_{1}\right.$, $\left.w_{2}, \ldots, w_{k}\right]$ is chosen as symmetric Dirichlet $D$ :

$$
\mathbf{w} \sim D(\zeta, \ldots, \zeta)
$$

(iii) Selection of prior information for component parameter vector $\boldsymbol{\theta}$ :

Location parameter $\mu$ is priorily drawn from normal distribution

$$
\mu \sim N\left(\mu \mid \xi, \kappa^{-1}\right) \quad \text { i.e. } p(\mu)=N\left(\mu \mid \xi, \kappa^{-1}\right)
$$

where $\xi$ is set as the midpoint of the interval of the data and $\kappa$ as a small multiple of $1 / R^{2}$, where $R$ is the length of the interval of data as in [Richardson \& Green, 1997].

The dispersion $\gamma$ is priorily drawn from an inverse gamma distribution with the hyperparameters $\alpha_{0}$ and $\beta_{0}$

$\gamma \sim I G\left(\gamma \mid \alpha_{0}, \beta_{0}\right) \quad$ i.e. $p(\gamma)=I G\left(\gamma \mid \alpha_{0}, \beta_{0}\right)$.

Prior distributions for the characteristic exponent $\alpha$ and the skewness $\beta$ are chosen as in [Salas-Gonzalez et al., 2009].

$$
\begin{aligned}
& p\left(\alpha_{j} \mid a\right)=\frac{1}{a}=\frac{1}{2}, \quad 0<\alpha_{j} \leq 2 \\
& p\left(\beta_{j} \mid b\right)=\frac{1}{b}=\frac{1}{2}, \quad-1 \leq \beta_{j} \leq 1 .
\end{aligned}
$$

\subsubsection{Updating $\mathbf{w}, \boldsymbol{\theta}, \mathbf{z}$}

For a conditionally hierarchical model the joint distribution of all variables can be expressed by using the Bayes' rule as

$$
\begin{aligned}
p\left(k_{0}, \zeta,\right. & \eta, k, w, z, \theta, y) \\
= & p(y \mid k, w, z, \theta, \eta) p(\theta \mid k, \eta) p(z \mid w, k) \\
& \times p(w \mid k, \zeta) p\left(k \mid k_{0}\right) p(\eta) p(\zeta) p\left(k_{0}\right)
\end{aligned}
$$

where $\eta=\left\{a, b, \alpha_{0}, \beta_{0}, \xi, \kappa\right\}$ and $k_{0}, \zeta, \eta$ are the hyperpriors for unknown variables $k, w, \theta$, respectively. The method for the estimation of component parameters in a Bayesian mixture model was given in [Richardson \& Green, 1997; Salas-Gonzalez et al., 2009]. The steps briefly presented below consist of the types of updates for a hierarchical stable mixture model:

(i) Updating the weight vector $\mathbf{w}$ :

The mixture proportions $\mathbf{w}$ have been updated by Gibbs sampling and the full conditional distribution of $\mathbf{w}$ is taken from a Dirichlet distribution as in the case of prior distribution for $\mathbf{w}$.

$$
\mathbf{w} \sim D\left(\zeta+n_{1}, \ldots, \zeta+n_{k}\right)
$$

where $n_{j}$ is the number of samples assigned to the component $j$.

(ii) Updating the component parameter vector $\boldsymbol{\theta}$ : $\alpha, \beta, \gamma, \mu$ parameters have been updated using Metropolis-Hastings algorithm [Rubinstein \& Kroese, 2011, p. 169] which is a powerful MCMC method.

\section{Metropolis-Hastings algorithm:}

(ii.1) At each iteration $t$, given $\boldsymbol{\theta}^{(t)}=\left[\boldsymbol{\theta}_{1}^{(t)}\right.$ $\left.\begin{array}{lll}\boldsymbol{\theta}_{2}^{(t)} & \cdots & \boldsymbol{\theta}_{k}^{(t)}\end{array}\right]$ where $\boldsymbol{\theta}_{j}^{(t)}=\left[\begin{array}{ll}\theta_{j 1}^{(t)} & \theta_{j 2}^{(t)}\end{array}\right.$ $\left.\begin{array}{ll}\theta_{j 3}^{(t)} & \theta_{j 4}^{(t)}\end{array}\right]$ then generate $\theta_{j m}^{\text {new }}$ for $m=1$, 2,3,4 from the proposal distributions $\theta_{j m}^{\text {new }} \sim q\left(\theta_{j m}^{\text {new }} \mid \theta_{j m}^{(t)}\right)$ where the proposal distributions $q$ have been described in Eqs. (8)-(11),

(ii.2) for each observation $y_{i}$, evaluate

$$
A_{\theta_{j m}}=\min \left\{1, \frac{\prod_{i}^{N} p\left(y_{i} \mid k, w_{j}, \theta_{j m}^{\text {new }}, \boldsymbol{\theta}_{j(-r)}\right)}{\prod_{i}^{N} p\left(y_{i} \mid k, w_{j}, \theta_{j m}^{(t)}, \boldsymbol{\theta}_{j(-r)}\right)} \frac{f\left(\theta_{j m}^{\text {new }} \mid \eta\right)}{f\left(\theta_{j m}^{(t)} \mid \eta\right)}\right\},
$$


where $\boldsymbol{\theta}_{j(-r)}$ consists of parameters $\theta_{j m}$ $m \neq r$ and $f$ is chosen as a uniform distribution for both characteristic exponent $\alpha$ and skewness $\beta$ while inverse gamma distribution (normal distribution) for dispersion $\gamma$ (location $\mu$ ) parameter, respectively,

(ii.3) generate $u \sim U(0,1)$ and then the proposed value $\theta_{j}^{\text {new }}$ is accepted (is not accepted) as a posterior value if $u \leq A_{\theta_{j}}$ (otherwise), respectively, i.e.

$$
\theta_{j m}^{(t+1)}= \begin{cases}\theta_{j m}^{\text {new }}, & \text { if } u \leq A_{\theta_{j m}} \\ \theta_{j m}^{(t)}, & \text { otherwise. }\end{cases}
$$

(iii) Updating the allocation $\mathbf{z}$ :

At every iteration $t$, the full conditional probability that the $i$ th observation being in the $j$ th cluster can be determined as

$$
\begin{aligned}
p\left(z_{i}\right. & \left.=j \mid \mathbf{z}_{-i}, \mathbf{y}, \boldsymbol{\theta}, \mathbf{w}, k\right) \\
& =p\left(z_{i}=j\right) \cdot p\left(\mathbf{y} \mid \mathbf{z}_{-i}, \boldsymbol{\theta}, \mathbf{w}, k\right)
\end{aligned}
$$

where $\mathbf{z}_{-i}$ is the cluster assignment for all observations except the $i$ th one i.e. $z_{-i}$ is the vector $\mathbf{z}$ without component $i$ denoted as $\mathbf{z}_{-i} \triangleq$ $\left(z_{1}, \ldots, z_{i-1}, z_{i+1}, \ldots, z_{N}\right)$ and $p\left(z_{i}=j\right)=w_{j}$ as described by Eq. (4).

\subsubsection{Estimating the number of mixtures $k$}

Reversible Jump Markov Chain Monte Carlo (RJMCMC) method moves among different number of finite normal (Gaussian) mixture models in [Green, 1995; Richardson \& Green, 1997; Gelman et al., 2013] and for non-normal mixture models in [McLachlan \& Peel, 2004]. The reversible jump algorithm given in [Green, 1995] has been summarized as follows:

\section{RJMCMC algorithm}

(i) Define the current state $x \triangleq(k, \mathbf{w}, \boldsymbol{\theta})$ which has $\operatorname{dimension} \operatorname{dim}(x)=5 k+1$ since $\operatorname{dim}(w)=$ $k, \operatorname{dim}(\theta)=4 k$ (for each mixture component there are four parameters $\alpha, \beta, \gamma, \mu$ to be estimated and therefore for $k$ number of mixtures totally $4 k$ parameters should be estimated).

(ii) Given a current state $x$, a Markov Chain move to the new state $x^{\prime}$ is achieved with probability $r_{m}\left(x^{\prime}\right)$. Since the dimension of the new state $x^{\prime}$ (i.e. $\operatorname{dim}\left(x^{\prime}\right)$ ) is different than the dimension of the previous state $x$ then the one-to-one deterministic function is defined as $\left(x^{\prime}, u^{\prime}\right)=g(x, u)$ by using additional random variable $u$ which is drawn from the jumping distribution $J(u)$ (independent of $x$ and $x^{\prime}$ ), where $\operatorname{dim}(x)+\operatorname{dim}(u)=\operatorname{dim}\left(x^{\prime}\right)+\operatorname{dim}\left(u^{\prime}\right)$,

(iii) then accept this Markov Chain move to the new state $x^{\prime}$ with probability $\min \left(1, A_{m}\left(x, x^{\prime}\right)\right)$ where

$$
A_{m}\left(x, x^{\prime}\right)=\frac{p\left(x^{\prime} \mid y\right) r_{m}\left(x^{\prime}\right)}{p(x \mid y) r_{m}(x) J(u)} \cdot\left|\frac{\partial g(x, u)}{\partial(x, u)}\right|
$$

where $r_{m}(x)$ is the probability of moving to $x$ when the current state is $x^{\prime}$ (i.e. a new model $x$ is proposed with probability $r_{m}(x)$ when the current state is $x^{\prime}$ ) and $|\cdot|$ denotes the determinant of the Jacobian matrix.

In $\mathrm{FM} \alpha \mathrm{S}$ distributions for each proposed number of mixtures $k$, new values for weights, location and dispersion parameters have been assigned by the method of splitting one mixture component into two, or combining two into one, namely, split/combine move while $\alpha$ and $\beta$ parameters have been assigned by setting the previous values as the new values. The details of split/combine move for estimating the number of mixtures $k$ in a mixture model of $\alpha$-stable distributions are given in [SalasGonzalez et al., 2009].

Remark (The Number of Clusters Known). If the number of clusters of the data is considered to be known or the data is desired to be modeled with a prespecified number of clusters then there is no need to estimate the number of mixtures $k$, therefore Secs. 2.2.1(i) and 2.2.3 could be skipped and hence computational costs could be reduced.

\section{Characterization of Distributions of Multiscrolls by the Parameters for the Finite Mixture of $\alpha$-Stable Distributions}

The dynamics of autonomous GCC [Suykens et al., 1997] are described by the following set of three differential equations

$$
\dot{x}=a[y-h(x)], \quad \dot{y}=x-y+z, \quad \dot{z}=-b y
$$


where $a$ and $b$ denote bifurcation parameters to avoid confusion with the parameters of $\alpha$-stable distribution and $h(x)$ is given as

$$
\begin{aligned}
h(x)= & m_{2 q-1} x+\frac{1}{2} \sum_{i=1}^{2 q-1}\left(m_{i-1}-m_{i}\right) \\
& \times\left(\left|x+c_{i}\right|-\left|x-c_{i}\right|\right)
\end{aligned}
$$

where $q$ determines the number of scrolls.

The data for various chaotic behavior have been simulated by numerically solving Eq. (18) using fifth-order Dormand-Prince algorithm with fixed step size. The first 100 data points have been ignored due to transients as done in the previous works [Günel, 2004; Savaci \& Güngör, 2012]. The parameters for the behaviors of limit cycle, double scroll, 3-scroll, and 5-scroll attractors have been chosen as in [Chua et al., 1986; Suykens et al., 1997].

In the following subsections, PDFs of the state $x$ of Chua's circuit have been estimated by $\mathrm{FM} \alpha \mathrm{S}$ distributions and the estimated parameter $w_{j}$ indicates the probability of data belonging to the cluster $j$; the estimated location parameter $\mu$ is the mean of the corresponding cluster; the estimated skewness parameter $\beta$ shows the inclination of the data about the location parameter $\mu$ and the estimated dispersion parameter $\gamma_{j}$ indicates the spread of the data about the location parameter which can be also considered as a measure of energy for the corresponding cluster.

The FM $\alpha$ S-distributions have been obtained for two cases:

Case I. When the number of clusters is unknown then RJMCMC algorithm has been applied to estimate the number of mixtures $k$.

Case II. When the number of clusters is known then the mixture proportions $\mathbf{w}$ and component parameters $(\alpha, \beta, \gamma, \mu)$ of $\alpha$-stable distributions have been estimated for a prespecified $k$.

In the sequel, the number of MCMC and RJMCMC iterations have been set as 10000 .

\subsection{Limit cycle}

The bifurcation parameters have been chosen as $a=$ $7, b=14.28, q=c=1$ and $m_{0}=-1 / 7, m_{1}=2 / 7$.

Case I. Unknown case: the initial value for the number of mixtures $k$ is arbitrarily set as $k_{0}=6$ and the number of mixtures $k$ has been estimated
Table 1. Estimated parameters for limit cycle.

\begin{tabular}{llll}
\hline Parameter & $\begin{array}{c}\text { \# of Clusters } \\
\text { Known }\end{array}$ & $\begin{array}{c}\text { \# of Clusters } \\
\text { Unknown }\end{array}$ \\
\hline$\left[\begin{array}{ll}w_{1} & w_{2}\end{array}\right]$ & {$\left[\begin{array}{ll}0.5171 & 0.4829\end{array}\right]$} & {$\left[\begin{array}{ll}0.5642 & 0.4358\end{array}\right]$} \\
{$\left[\begin{array}{ll}\alpha_{1} & \alpha_{2}\end{array}\right]$} & {$\left[\begin{array}{ll}0.884 & 0.901\end{array}\right]$} & {$\left[\begin{array}{lll}1.0058 & 0.9159\end{array}\right]$} \\
{$\left[\begin{array}{ll}\beta_{1} & \beta_{2}\end{array}\right]$} & {$\left[\begin{array}{ll}1.00 & -1.00\end{array}\right]$} & {$\left[\begin{array}{lll}1.00 & -1.00\end{array}\right]$} \\
{$\left[\begin{array}{ll}\gamma_{1} & \gamma_{2}\end{array}\right]$} & {$\left[\begin{array}{ll}0.104 & 0.089\end{array}\right]$} & {$\left[\begin{array}{ll}0.1048 & 0.058\end{array}\right]$} \\
{$\left[\begin{array}{ll}\mu_{1} & \mu_{2}\end{array}\right]$} & {$\left[\begin{array}{ll}0.948 & 1.989\end{array}\right]$} & {$\left[\begin{array}{ll}0.9515 & 1.989\end{array}\right]$} \\
\hline
\end{tabular}

by the algorithm given in Sec. 2.2.3 and obtained as $k=2$ for 9932 iterations. Hence the distribution can be modeled by the mixture of two $\alpha$-stable densities expressed as

$$
p(x)=\sum_{j=1}^{2} w_{j} \cdot f_{j}\left(\alpha_{j}, \beta_{j}, \gamma_{j}, \mu_{j}\right)
$$

where the weight vector $\mathbf{w}=\left[\begin{array}{ll}w_{1} & w_{2}\end{array}\right]$ and component parameters have been estimated as shown in Table 1.

Case II. Known case: the number of clusters has been prespecified as $k=2$ and the estimated parameters have been listed in Table 1. As seen from Table 1 the estimated parameters are close to each other for both cases.

In Fig. 1, the estimated mixture model of $\alpha$ stable distributions for both cases have been shown and both estimated $\mathrm{FM} \alpha \mathrm{S}$ distributions have fitted well to the histogram of the data obtained from the limit cycle.

Remark (Speed of the Trajectory versus Extrema of $\mathrm{FM} \alpha \mathrm{S}$ Distributions). In Fig. 2 the trajectory of

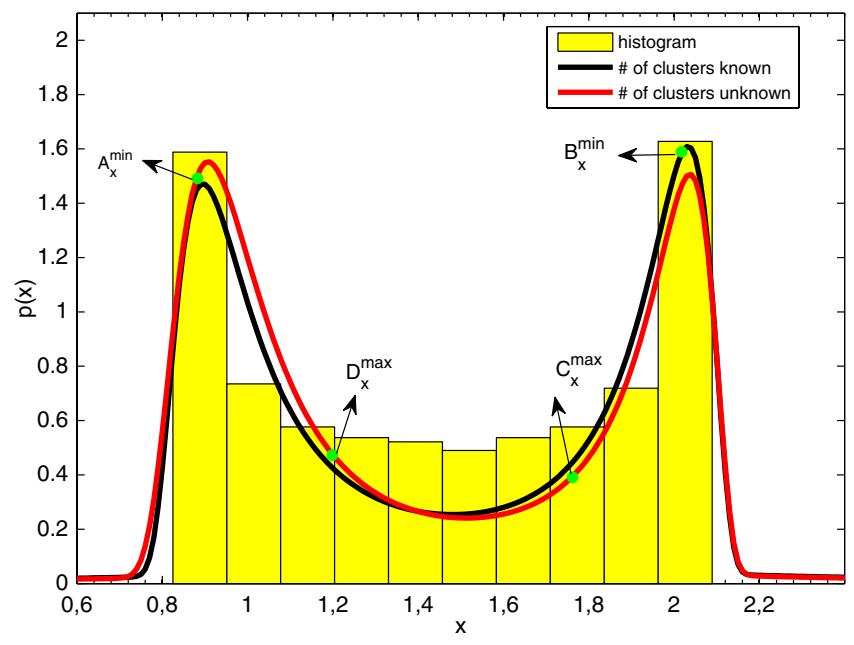

Fig. 1. Estimated FM $\alpha \mathrm{S}$ distributions for limit cycle, the number of mixtures is known (black line), and the number of mixtures is unknown (red line). 


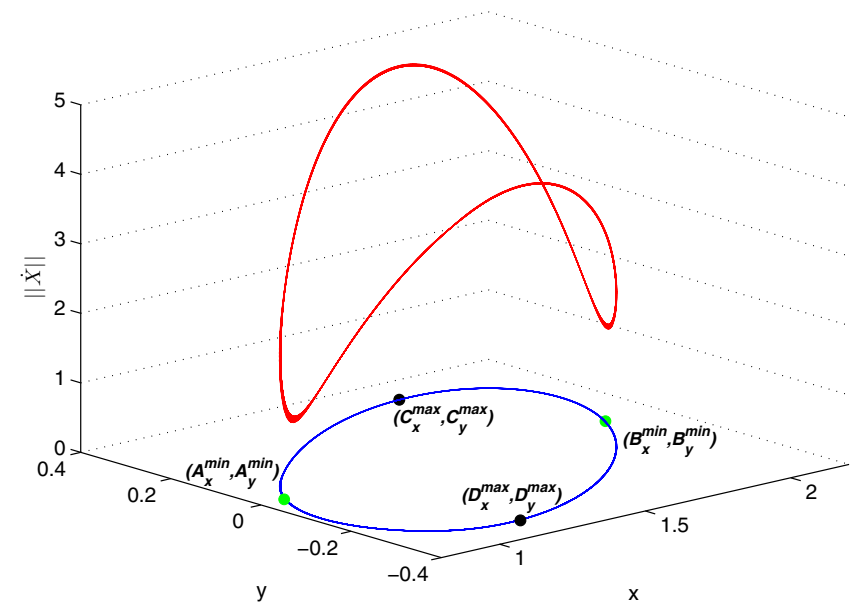

Fig. 2. The phase portrait of limit cycle (blue line) and $\|\dot{\mathbf{X}}\|$ (red line).

the system Eq. (18) is shown with blue-line and the corresponding Euclidean norm of $\dot{\mathbf{X}} \triangleq\left[\begin{array}{lll}\dot{x} & \dot{y} & \dot{z}\end{array}\right]$ is shown with red-line. $\mathbf{A}_{\min }$ and $\mathbf{B}_{\text {min }}$ correspond to the points at which the speed of the trajectory is minimum (i.e. minimum of $\|\dot{\mathbf{X}}\|$ occur at these points) whereas $\mathbf{C}_{\max }$ and $\mathbf{D}_{\max }$ correspond to the points at which Euclidean norm $\|\dot{\mathbf{X}}\|$ are maximum. From Figs. 1 and 2, it can be observed that when the trajectory slows down, it spends longer time nearby $\mathbf{A}_{\text {min }}$ and $\mathbf{B}_{\min }$ and consequently, the local maxima of the estimated $\mathrm{FM} \alpha \mathrm{S}$ distributions occur at these points. The local minimum of the estimated $\mathrm{FM} \alpha \mathrm{S}$ distributions occurs in the interval of $\mathbf{C}_{x}^{\max }$ and $\mathbf{D}_{x}^{\max }$ where $\|\dot{\mathbf{X}}\|$ increase (i.e. the trajectory moves faster).

\subsection{Double scroll}

The bifurcation parameters have been chosen as $a=$ $9, b=14.28, q=c=1$ and $m_{0}=-1 / 7, m_{1}=2 / 7$.

Case I. Unknown case: the number of mixtures $k$ has been estimated as $k=4$ for 8135 iterations. Hence the observed data of the double scroll can be modeled by the mixture of four $\alpha$-stable densities expressed as

$$
\sum_{j=1}^{4} w_{j} \cdot f_{j}\left(\alpha_{j}, \beta_{j}, \gamma_{j}, \mu_{j}\right)
$$

where the weight vector $\mathbf{w}=\left[\begin{array}{lll}w_{1} & \cdots & w_{4}\end{array}\right]$ and component vectors $\boldsymbol{\alpha}=\left[\begin{array}{lll}\alpha_{1} & \cdots & \alpha_{4}\end{array}\right], \boldsymbol{\beta}=$ $\left[\begin{array}{lll}\beta_{1} & \cdots & \beta_{4}\end{array}\right], \quad \gamma=\left[\begin{array}{lll}\gamma_{1} & \cdots & \gamma_{4}\end{array}\right]$ and $\boldsymbol{\mu}=$ $\left[\begin{array}{lll}\mu_{1} & \cdots & \mu_{4}\end{array}\right]$ have been estimated as shown in Table 2.

Case II. Known case: the number of clusters has been prespecified as $k=4$ and the estimated parameters have been listed in Table 2. As seen from Table 2, for both cases the algorithm has estimated the parameters as reasonably close to each other.

The estimated mixture stable model for double scroll for known case and unknown case has been shown in Fig. 3.

Remark (Speed of the Trajectory versus Extrema of $\mathrm{FM} \alpha \mathrm{S}$ Distributions). In Fig. 4 the trajectory of the system is shown where the green points correspond to the points at which $\|\dot{\mathbf{X}}\|$ are relatively small and larger values of $\|\dot{\mathbf{X}}\|$ occur at red points. From Figs. 3 and 4, it can be observed that when the trajectory slows down it spends longer time nearby green points, consequently, the local maxima of the estimated $\mathrm{FM} \alpha \mathrm{S}$ distributions occur at these points. The local minima of the estimated $\mathrm{FM} \alpha \mathrm{S}$ distributions occur in the interval of red points at which $\|\dot{\mathbf{X}}\|$ are relatively high (i.e. the trajectory moves faster). As the simulation results show that the speed of the trajectory slows down nearby the boundaries of the state $x$ hence the occurrence of these points increases which implies heavy-tailness at the boundaries of state $x$.

\subsection{3-scroll}

The bifurcation parameters have been chosen as $a=9, b=14.28, q=2$ and $m_{0}=0.9 / 7, m_{1}=$ $-3 / 7, m_{2}=3.5 / 7, m_{3}=-2.4 / 7, c_{1}=1, c_{2}=$ $2.15, c_{3}=4$.

Table 2. Estimated parameters for double scroll.

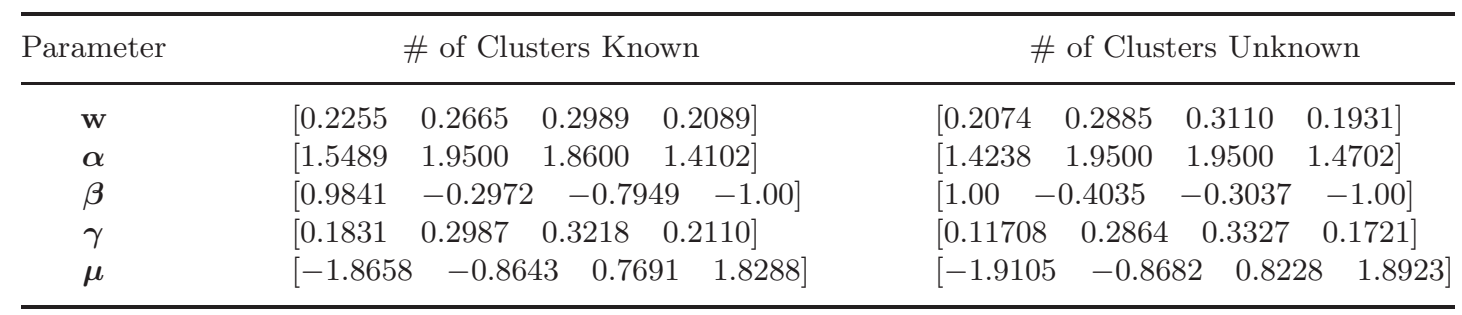




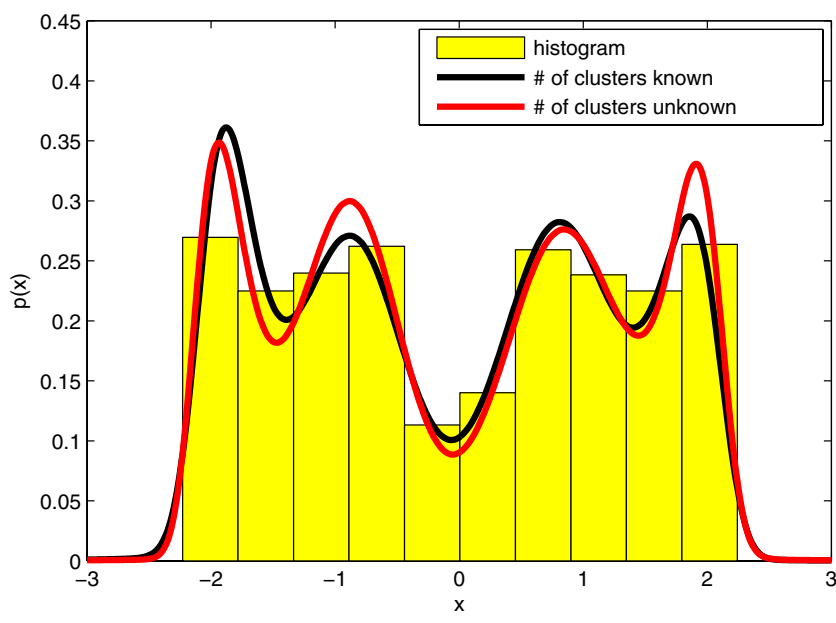

Fig. 3. Estimated FM $\alpha \mathrm{S}$ distributions for double scroll, the number of mixtures is known (black line), and the number of mixtures is unknown (red line).

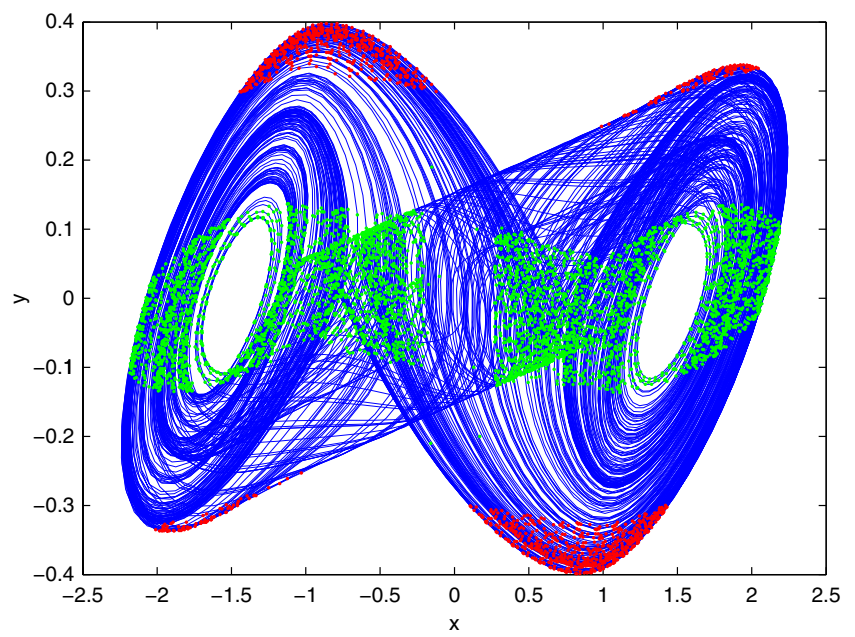

Fig. 4. 2D phase portrait for double scroll (at green points the trajectory slows down and at red points the trajectory moves faster).

Case I. Unknown case: $k_{0}=11$ has been chosen as the initial value and the number of mixtures $k$ has been estimated as $k=3$ for 8135 iterations. The observed data can be modeled by the mixture of three $\alpha$-stable densities expressed as

$$
\sum_{j=1}^{3} w_{j} \cdot f_{j}\left(\alpha_{j}, \beta_{j}, \gamma_{j}, \mu_{j}\right)
$$

where the weight vector $\mathbf{w}=\left[\begin{array}{lll}w_{1} & \cdots & w_{3}\end{array}\right]$ and component vectors $\boldsymbol{\alpha}=\left[\begin{array}{lll}\alpha_{1} & \cdots & \alpha_{3}\end{array}\right], \boldsymbol{\beta}=$ $\left[\begin{array}{lll}\beta_{1} & \cdots & \beta_{3}\end{array}\right], \gamma=\left[\begin{array}{lll}\gamma_{1} & \cdots & \gamma_{3}\end{array}\right]$ and $\boldsymbol{\mu}=$ $\left[\begin{array}{lll}\mu_{1} & \cdots & \mu_{3}\end{array}\right]$ have been estimated as shown in Table 3.

Case II. Known case: the number of clusters has been prespecified as $k=3$ and the estimated parameters have been listed in Table 3 .

The estimated mixture stable model for 3-scroll attractors for known and unknown cases has been shown in Fig. 6.

\subsection{5-scroll}

The bifurcation parameters have been chosen as $a=9, b=14.28, q=3$ and $m_{0}=0.9 / 7, m_{1}=$ $-3 / 7, m_{2}=3.5 / 7, m_{3}=-2.7 / 7, m_{4}=4 / 7, m_{5}=$ $-2.4 / 7, c_{1}=1 ; c_{2}=2.15, c_{3}=3.6, c_{4}=6.2, c_{5}=9$.

Case I. Unknown case: the number of mixtures $k$ has been estimated as $k=5$ for 5486 iterations. Hence the observation can be modeled by the mixture of five $\alpha$-stable densities expressed as

$$
\sum_{j=1}^{5} w_{j} \cdot f_{j}\left(\alpha_{j}, \beta_{j}, \gamma_{j}, \mu_{j}\right)
$$

where the weight vector $\mathbf{w}=\left[\begin{array}{lll}w_{1} & \cdots & w_{5}\end{array}\right]$ and component vectors $\boldsymbol{\alpha}=\left[\begin{array}{lll}\alpha_{1} & \cdots & \alpha_{5}\end{array}\right], \boldsymbol{\beta}=$ $\left[\begin{array}{lll}\beta_{1} & \cdots & \beta_{5}\end{array}\right], \quad \gamma=\left[\begin{array}{lll}\gamma_{1} & \cdots & \gamma_{5}\end{array}\right]$ and $\boldsymbol{\mu}=$ $\left[\begin{array}{lll}\mu_{1} & \cdots & \mu_{5}\end{array}\right]$ have been estimated as shown in Table 4.

Case II. Known case: the number of clusters has been prespecified as $k=5$ and the estimated parameters have been listed in Table 4. As seen from Table 4 for both cases as in the previous chaotic behaviors, the algorithm has estimated the

Table 3. Estimated parameters for 3-scroll attractor.

\begin{tabular}{cllll}
\hline Parameter & \multicolumn{3}{c}{ \# of Clusters Known } & \multicolumn{3}{c}{ \# of Clusters Unknown } \\
\hline $\mathbf{w}$ & {$\left[\begin{array}{lllll}0.3771 & 0.2820 & 0.3409\end{array}\right]$} & {$\left[\begin{array}{llll}0.3634 & 0.2952 & 0.3414\end{array}\right]$} \\
$\boldsymbol{\alpha}$ & {$\left[\begin{array}{llll}1.9500 & 1.9500 & 1.9500\end{array}\right]$} & {$\left[\begin{array}{llll}1.9500 & 1.9500 & 1.9500\end{array}\right]$} \\
$\boldsymbol{\beta}$ & {$\left[\begin{array}{llll}0.6361 & -0.1338 & -0.5371\end{array}\right]$} & {$\left[\begin{array}{lll}0.0968 & -0.6386 & -0.6994\end{array}\right]$} \\
$\boldsymbol{\gamma}$ & {$\left[\begin{array}{llll}0.2628 & 0.7608 & 0.2745\end{array}\right]$} & {$\left[\begin{array}{llll}0.2531 & 0.8051 & 0.2674\end{array}\right]$} \\
$\boldsymbol{\mu}$ & {$\left[\begin{array}{lllll}-2.9017 & 0.0893 & 2.8817\end{array}\right]$} & {$\left[\begin{array}{llll}-2.8811 & 0.2952 & 2.8832\end{array}\right]$} \\
\hline
\end{tabular}




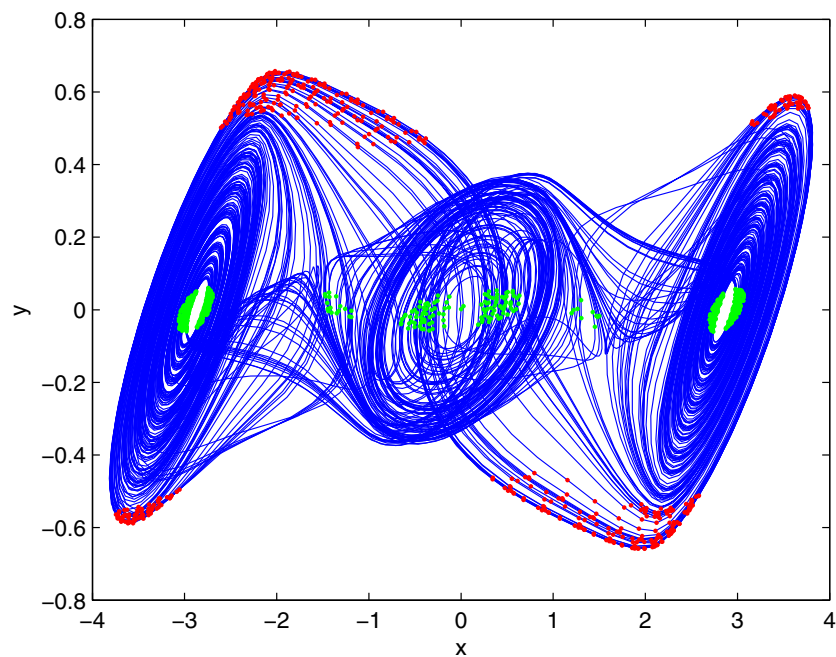

Fig. 5. 2D phase portrait for 3-scroll attractors (at green points the trajectory slows down and at red points the trajectory moves faster).

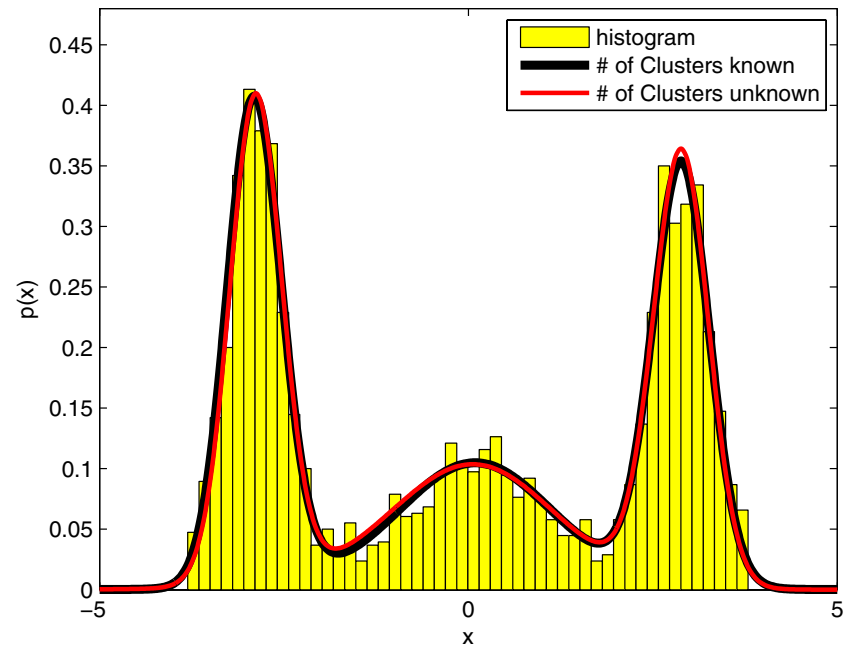

Fig. 6. Estimated $\mathrm{FM} \alpha \mathrm{S}$ distributions for 3-scroll attractors, the number of mixtures is known (black line), and the number of mixtures is unknown (red line).

Table 4. Estimated parameters for 5-scroll attractor

\begin{tabular}{|c|c|c|}
\hline Parameter & \# of Clusters Known & \# of Clusters Unknown \\
\hline $\mathbf{w}$ & {$\left[\begin{array}{lllll}0.1586 & 0.2297 & 0.3594 & 0.1486 & 0.1037\end{array}\right]$} & {$\left[\begin{array}{lllll}0.1619 & 0.2317 & 0.3360 & 0.1549 & 0.1154\end{array}\right]$} \\
\hline$\alpha$ & {$\left[\begin{array}{lllll}1.8164 & 1.8141 & 1.7615 & 1.8264 & 1.5654\end{array}\right]$} & {$\left[\begin{array}{lllll}1.8067 & 1.9500 & 1.9500 & 1.9500 & 1.5347\end{array}\right]$} \\
\hline $\boldsymbol{\beta}$ & {$\left[\begin{array}{lllll}0.5248 & -0.1657 & -0.2943 & 0.7923 & -1.00\end{array}\right]$} & {$\left[\begin{array}{lllll}1.00 & -0.9158 & -0.2939 & 0.0837 & -1.00\end{array}\right]$} \\
\hline$\gamma$ & {$\left[\begin{array}{lllll}0.4371 & 0.4392 & 0.6278 & 0.3964 & 0.5402\end{array}\right]$} & {$\left[\begin{array}{lllll}0.4131 & 0.4623 & 0.6182 & 0.4035 & 5257\end{array}\right]$} \\
\hline$\mu$ & {$\left[\begin{array}{lllll}-7.3182 & -2.8775 & 0.0769 & 2.9361 & 7.4007\end{array}\right]$} & {$\left[\begin{array}{lllll}-7.3852 & -2.9087 & 0.0081 & 2.9019 & 7.4199\end{array}\right]$} \\
\hline
\end{tabular}

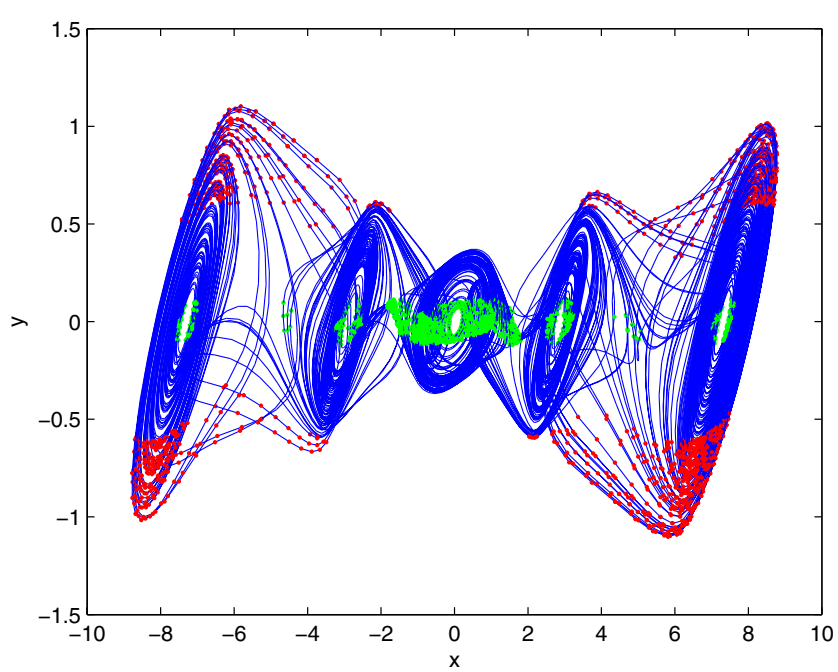

Fig. 7. 2D phase portrait for 5 -scroll attractors (at green points the trajectory slows down and at red points the trajectory moves faster).

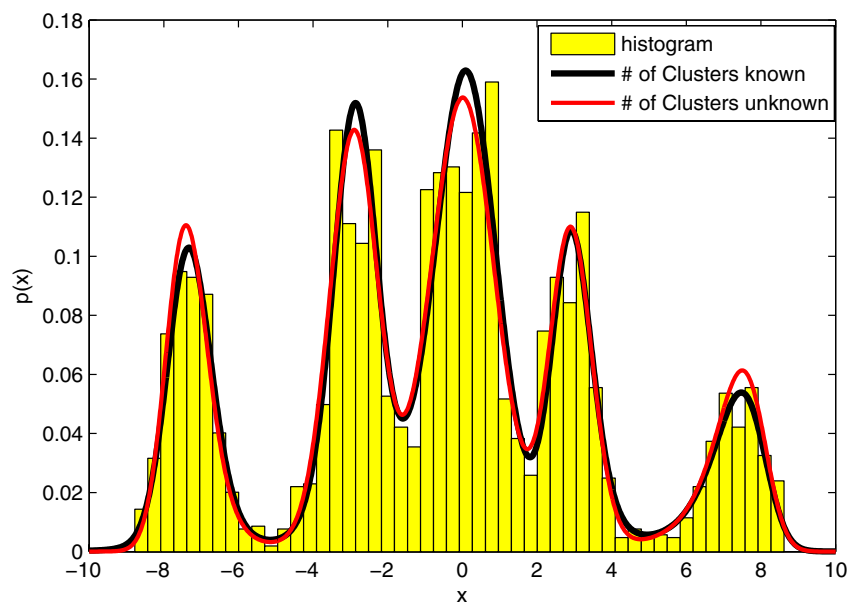

Fig. 8. Estimated $\mathrm{FM} \alpha \mathrm{S}$ distributions for 5-scroll attractors, the number of mixtures is known (black line), and the number of mixtures is unknown (red line). 


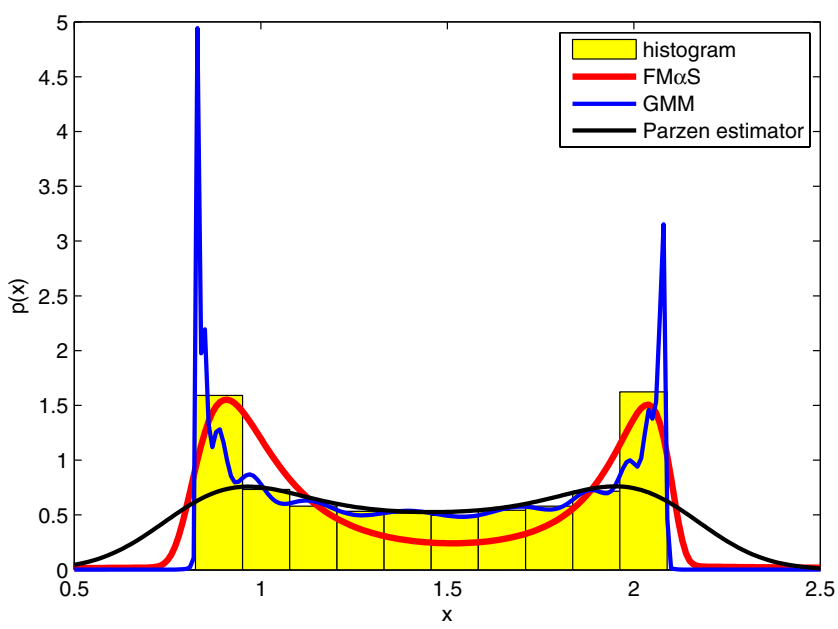

Fig. 9. Comparison of estimated $\mathrm{FM} \alpha \mathrm{S}$ distribution with GMM and Parzen estimator for limit cycle.

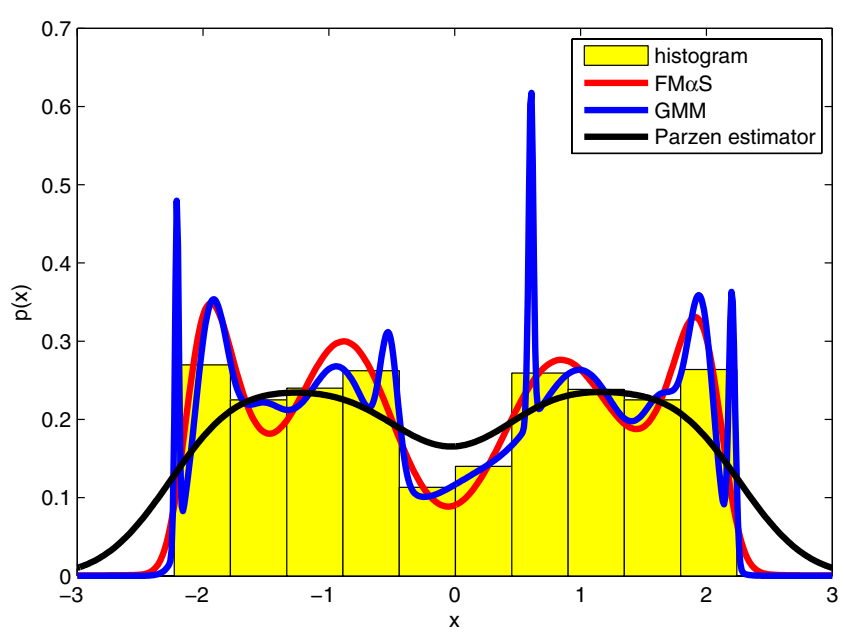

Fig. 10. Comparison of estimated FM $\alpha \mathrm{S}$ distribution with GMM and Parzen estimator for double scroll attractor.

parameters as reasonably close to each other which proves the strength of the RJMCMC algorithm.

Figure 8 shows the mixture model for the estimated parameters given in Table 4. As seen from Fig. 8, the estimated FM $\alpha \mathrm{S}$ distributions for the known and unknown cases have been fitted to the

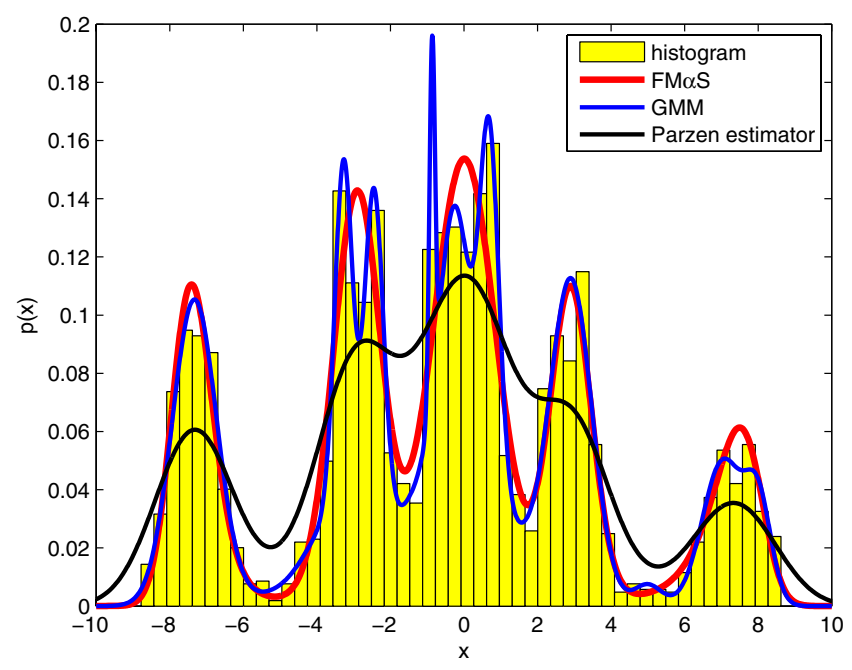

Fig. 11. Comparison of estimated $\mathrm{FM} \alpha \mathrm{S}$ distribution with GMM and Parzen estimator for 5-scroll attractor.

histogram of data. It has been also observed that the location parameters of the estimated $\mathrm{FM} \alpha \mathrm{S}$ distributions correspond to points at which $\|\dot{\mathbf{X}}\|$ are relatively small.

\subsection{FMoS model versus GMM and Parzen estimator}

Figures 9-11 show the comparison of $\mathrm{FM} \alpha \mathrm{S}$ model with GMM and Parzen estimator. Gaussian Mixture Model (GMM) which fits the histogram obtained from the corresponding attractors has been determined by using Akaike Information Criterion (AIC) [Webb \& Copsey, 2011] and the number of components has been found as listed in Table 5 .

The statistical Bhattacharyya distance [Bhattacharyya, 1946; Kailath, 1967] between the histograms and the models has been calculated for comparison with the models. As seen from Table 5, $\mathrm{FM} \alpha \mathrm{S}$ yields smaller error and less number of mixture components. From this comparison it can be concluded that when the number of scrolls gets less which results in more heaviness in the tails then

Table 5. Comparison of the distance measure.

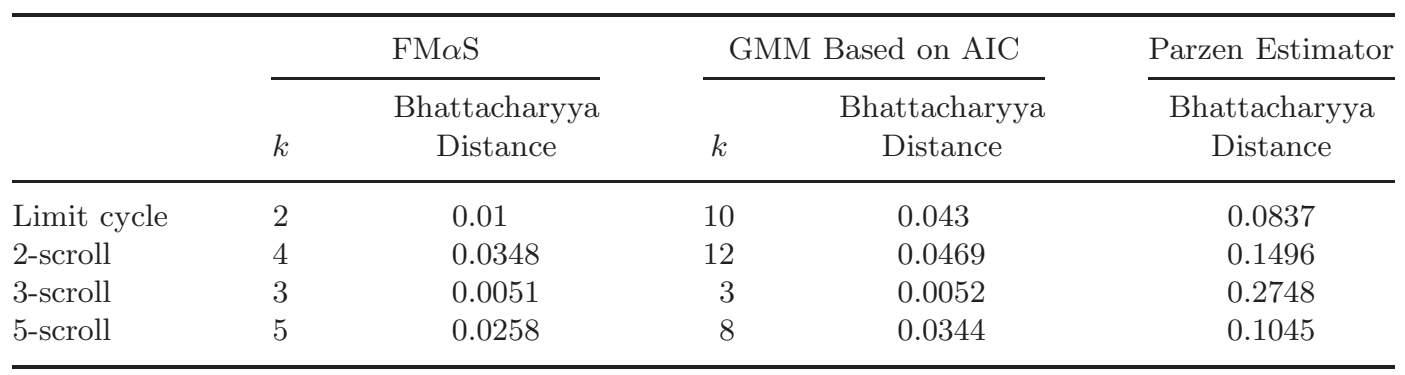


the $\mathrm{FM} \alpha \mathrm{S}$ is more accurate for modeling and as the number of scrolls increase (resulting in less heaviness in the tails) the accuracy of GMM model approaches that of $\mathrm{FM} \alpha \mathrm{S}$ model.

\section{Conclusion and Future Work}

In this paper, $\mathrm{FM} \alpha \mathrm{S}$ distributions have been used to model the state PDFs of GCC. The parameters of the distributions have been estimated by the Bayesian algorithm. When GCC is considered as black box from which the data are obtained then the number of clusters cannot be known beforehand and in this case RJMCMC algorithm has successfully estimated the true number of mixtures " $k$ " value and the resulting $\mathrm{FM} \alpha \mathrm{S}$ distributions have well fitted to the histogram of the simulated data. On the other hand, when the state space model of GCC is known then we can a priori determine the number of clusters which might be greater than the number of scrolls for GCC then the Bayesian algorithm without using RJMCMC method still achieves true $\mathrm{FM} \alpha \mathrm{S}$ distributions which also well fit to the histogram. Actually, the prior knowledge is to know the state space model of GCC from which the data are obtained. When the state space model is known the local minima of the vector field $\|\dot{\mathbf{X}}\|$ can be found either from simulations or analytically (if possible). These local minima of $\|\dot{\mathbf{X}}\|$ correspond to the location of clusters. Therefore, when it is possible to determine the number of clusters from the dynamics then modeling with known number of mixtures (Secs. 2.2.1-2.2.2) can be preferred to reduce the computational costs because avoiding RJMCMC algorithm given in Sec. 2.2.3 minimizes the complexity.

The FM $\alpha$ S distributions of GCC have well fitted not only to the histogram but also to the estimated PDFs using B-spline functions in [Savaci \& Güngör, 2012] and it has been observed in Sec. 3.5 that $\mathrm{FM} \alpha \mathrm{S}$ models have yielded smaller errors compared to the GMM. It has also been observed that the estimated values of some impulsiveness parameters $\alpha$ increase as the number of scrolls increases implying the decrease of heavy-tailness in the FM $\alpha \mathrm{S}$ mixture model. For all the estimated $\mathrm{FM} \alpha \mathrm{S}$ distributions in Sec. 3 the negative (positive) skewness parameter $\beta$ has indicated thicker left (right) tail and the estimated location parameters represent the mean value for each cluster.

$\mathrm{FM} \alpha \mathrm{S}$ distributions might become substantial in the determination of the solution of FPK equation. The determination of the stationary density for the Fokker-Planck-Kolmogorov (FPK) equation is a crucial problem [Lasota \& Mackey, 1994]. Multi-Gaussian Closure method [Er, 1998] in which the stationary probability density functions (PDFs) are assumed to be the weighted average of the Gaussian densities have been used to find the stationary density whereas in [Gunel \& Savaci, 2005a; Günel \& Savacı, 2006] the stationary density has been represented by compactly supported multivariable polynomials (CSP). The evolution of the PDF has also received interest recently for FPK of power systems in the analysis of stochastic power system stability [Wang \& Crow, 2013]. As a future work, $\mathrm{FM} \alpha \mathrm{S}$ distributions can be used to determine the approximate stationary solution of the FPK equation in power grids.

Controlling the shape of the PDF has received growing interest [Wang et al., 2013; Sun, 2006] and since $\mathrm{FM} \alpha \mathrm{S}$ distributions represent more general class of distributions, then $\mathrm{FM} \alpha \mathrm{S}$ distributions might be a more convenient model in the analysis of controlling the PDF of the dynamical system.

\section{Acknowledgments}

The numerical calculations reported in this paper were performed at TUBITAK ULAKBIM, High Performance and Grid Computing Center (TRUBA Resources).

The authors would also like to thank the anonymous reviewers for their valuable comments and suggestions to improve the final version of the paper.

\section{References}

Andrieu, C., Djurić, P. \& Doucet, A. [2001] "Model selection by MCMC computation," Sign. Process. 81, 19-37.

Bhattacharyya, A. [1946] "On a measure of divergence between two multinomial populations," Sankhyā: The Indian J. Statist. 7, 401-406.

Casarin, R. [2004] "Bayesian inference for mixtures of stable distributions," Working paper No. 0428, Cahier du CEREMADE, University Paris IX.

Chua, L. O., Komuro, M. \& Matsumoto, T. [1986] "The double scroll family," IEEE Trans. Circuits Syst. 33, 1072-1118.

Er, G.-K. [1998] "Multi-Gaussian closure method for randomly excited non-linear systems," Int. J. NonLin. Mech. 33, 201-214. 
Gelman, A., Carlin, J. B., Stern, H. S., Dunson, D. B., Vehtari, A. \& Rubin, D. B. [2013] Bayesian Data Analysis (CRC Press).

Green, P. J. [1995] "Reversible jump Markov chain Monte Carlo computation and Bayesian model determination," Biometrika 82, 711-732.

Günel, S. [2004] "An information theoretic approach to nonlinear control systems," PhD thesis, Dokuz Eylül University, Turkey.

Gunel, S. \& Savaci, F. A. [2005a] "Approximate stationary density of the nonlinear dynamical systems excited with white noise," IEEE Int. Symp. Circuits and Systems, 2005. ISCAS 2005 (IEEE), pp. 48994902.

Günel, S. \& Savaci, F. A. [2005b] 13th Int. Workshop on Nonlinear Dynamics of Electronic Systems, Postdam, Germany.

Günel, S. \& Savac1, F. A. [2006] "Determination of the stationary state densities of the stochastic nonlinear dynamical systems," Int. J. Engin. Sci. 44, 14321447.

Janicki, A. \& Weron, A. [1993] Simulation and Chaotic Behavior of Alpha-Stable Stochastic Processes, Vol. 178 (CRC Press).

Kailath, T. [1967] "The divergence and Bhattacharyya distance measures in signal selection," IEEE Trans. Commun. Technol. 15, 52-60.

Kuruoglu, E. E. [2001] "Density parameter estimation of skewed $\alpha$-stable distributions," IEEE Trans. Sign. Process. 49, 2192-2201.

Lasota, A. \& Mackey, M. C. [1994] Chaos, Fractals, and Noise: Stochastic Aspects of Dynamics, Vol. 97 (Springer).

Lombardi, M. J. [2007] "Bayesian inference for $\alpha$-stable distributions: A random walk MCMC approach," Comput. Statist. Data Anal. 51, 2688-2700.

Ma, X. \& Nikias, C. L. [1995] "Parameter estimation and blind channel identification in impulsive signal environments," IEEE Trans. Sign. Process. 43, 28842897.

McLachlan, G. \& Peel, D. [2004] Finite Mixture Models (John Wiley \& Sons).

Mei, S., Cao, M. \& Zhang, X. [2011] Power Grid Complexity (Springer).

Nikias, C. L. \& Shao, M. [1995] Signal Processing with $\alpha$-Stable Distributions and Applications (WileyInterscience).
Nolan, J. P. [1997] "Numerical calculation of stable densities and distribution functions," Commun. Statist. Stoch. Mod. 13, 759-774.

Richardson, S. \& Green, P. J. [1997] "On Bayesian analysis of mixtures with an unknown number of components (with discussion)," J. Roy. Statist. Soc.: Ser. B (Statist. Method.) 59, 731-792.

Rubinstein, R. Y. \& Kroese, D. P. [2011] Simulation and the Monte Carlo Method, Vol. 707 (John Wiley \& Sons).

Salas-Gonzalez, D., Kuruoglu, E. E. \& Ruiz, D. P. [2009] "Finite mixture of $\alpha$-stable distributions," Dig. Sign. Process. 19, 250-264.

Samoradnitsky, G. \& Taqqu, M. S. [1994] Stable NonGaussian Random Processes: Stochastic Models with Infinite Variance, Vol. 1 (CRC Press).

Savaci, F. \& Güngör, M. [2012] "Estimating probability density functions and entropies of Chua's circuit using B-spline functions," Int. J. Bifurcation and Chaos 22, 1250107-1-13.

Scott, D. W. [2009] Multivariate Density Estimation: Theory, Practice, and Visualization, Vol. 383 (John Wiley \& Sons).

Sun, J.-Q. [2006] Stochastic Dynamics and Control, Vol. 4 (Elsevier).

Suykens, J. A. \& Vandewalle, J. [1993] "Generation of $n$ double scrolls $(n=1,2,3,4$,$) ," IEEE Trans. Circuits$ Syst.-I: Fund. Th. Appl. 40, 861-867.

Suykens, J. A., Huang, A. \& Chua, L. O. [1997] "A family of $n$-scroll attractors from a generalized Chua's circuit," Archiv fur Elektronik und Ubertragungstechnik 51, 131-137.

Wang, K. \& Crow, M. L. [2013] "The Fokker-Planck equation for power system stability probability density function evolution," IEEE Trans. Power Syst. 28, 2994-3001.

Wang, L., Qian, F. \& Liu, J. [2013] "The pdf shape control of the state variable for a class of stochastic systems," Int. J. Syst. Sci., 1-9.

Webb, A. R. \& Copsey, K. D. [2011] Statistical Pattern Recognition (John Wiley \& Sons).

Zong, Z. [2006] Information-Theoretic Methods for Estimating of Complicated Probability Distributions, Vol. 207 (Elsevier). 
Copyright of International Journal of Bifurcation \& Chaos in Applied Sciences \& Engineering is the property of World Scientific Publishing Company and its content may not be copied or emailed to multiple sites or posted to a listserv without the copyright holder's express written permission. However, users may print, download, or email articles for individual use. 